\title{
Elimination of formate production in Clostridium thermocellum
}

\author{
Thomas Rydzak $^{1,2} \cdot$ Lee R. Lynd ${ }^{2,3} \cdot$ Adam M. Guss $^{1,2}{ }^{(1)}$
}

Received: 9 March 2015 / Accepted: 15 June 2015 / Published online: 11 July 2015

(C) The Author(s) 2015. This article is published with open access at Springerlink.com

\begin{abstract}
The ability of Clostridium thermocellum to rapidly degrade cellulose and ferment resulting hydrolysis products into ethanol makes it a promising platform organism for cellulosic biofuel production via consolidated bioprocessing. Currently, however, ethanol yield is far below theoretical maximum due to branched product pathways that divert carbon and electrons towards formate, $\mathrm{H}_{2}$, lactate, acetate, and secreted amino acids. To redirect carbon and electron flux away from formate, genes encoding pyruvate:formate lyase $(p f B)$ and PFL-activating enzyme ( $p f A)$ were deleted. Formate production in the
\end{abstract}

This manuscript has been authored by UT-Battelle, LLC under Contract No. DE-AC05-00OR22725 with the U.S. Department of Energy. The United States Government retains and the publisher, by accepting the article for publication, acknowledges that the United States Government retains a non-exclusive, paid-up, irrevocable, world-wide license to publish or reproduce the published form of this manuscript, or allow others to do so, for United States Government purposes. The Department of Energy will provide public access to these results of federally sponsored research in accordance with the DOE Public Access Plan (http:// energy.gov/downloads/doe-public-access-plan).

Electronic supplementary material The online version of this article (doi:10.1007/s10295-015-1644-3) contains supplementary material, which is available to authorized users.

Adam M. Guss

gussam@ornl.gov

1 Biosciences Division, Oak Ridge National Laboratory, Oak Ridge, TN, USA

2 BioEnergy Science Center, Oak Ridge National Laboratory, One Bethel Valley Road Oak Ridge, Tennessee 37831-6038, USA

3 Thayer School of Engineering at Dartmouth College, Hanover, NH, USA resulting $\Delta p f$ strain was eliminated and acetate production decreased by $50 \%$ on both complex and defined medium. The growth rate of the $\Delta p f l$ strain decreased by 2.9 -fold on defined medium and biphasic growth was observed on complex medium. Supplementation of defined medium with $2 \mathrm{mM}$ formate restored $\Delta p f$ growth rate to $80 \%$ of the parent strain. The role of $p f$ in metabolic engineering strategies and $\mathrm{C}_{1}$ metabolism is discussed.

Keywords Cellulosic ethanol $\cdot$ Clostridium thermocellum Pyruvate:formate lyase $\cdot$ Metabolic engineering $\cdot \mathrm{C} 1$ metabolism

\section{Introduction}

Growing global energy demands, rural economic development, the volatile cost of fossil fuels, and environmental concerns have prompted research into the development of sustainable and environmentally benign energy sources. Biofuels provide a promising alternative to petroleumderived fuels for transportation, one of the largest and fastest growing energy sectors [6]. Currently, bioethanol is a leading candidate that can be used with current technologies as a fuel supplement or replacement. A number of strains that produce ethanol at high yield and titer (e.g., Saccharomyces cerevisiae or Zymomonas mobilis) are available for industrial bioethanol production, but require monosaccharides or disaccharides typically derived from food/feed sources (e.g., corn, beets, sugarcane). These sugars can also be generated through chemical or enzymatic hydrolysis of highly abundant lignocellulosic biomass. However, consolidated bioprocessing (CBP), featuring one-step processing without added enzymes, has potential for lower costs as compared to processes that involve 
Fig. 1 Fermentation pathways in $C$. thermocellum. $F d$ ferredoxin; $L D H$ lactate dehydrogenase; $P F O R$ pyruvate: Fd oxidoreductase; $P F L$ pyruvate: formate lyase; $P T A /$ $A C K$ phosphotransacetylase/ acetate kinase; AldH/ADH aldehyde dehydrogenase/ alcohol dehydrogenase; $\mathrm{H}_{2}$ ases hydrogenases including echtype Fd-dependent hydrogenase and bifurcating hydrogenases. Oxidoreductases include NADH dependent reduced ferredoxin: $\mathrm{NADP}^{+}$oxidoreductase (NfnAB) and NADH:Fd oxidoreductase (RNF)

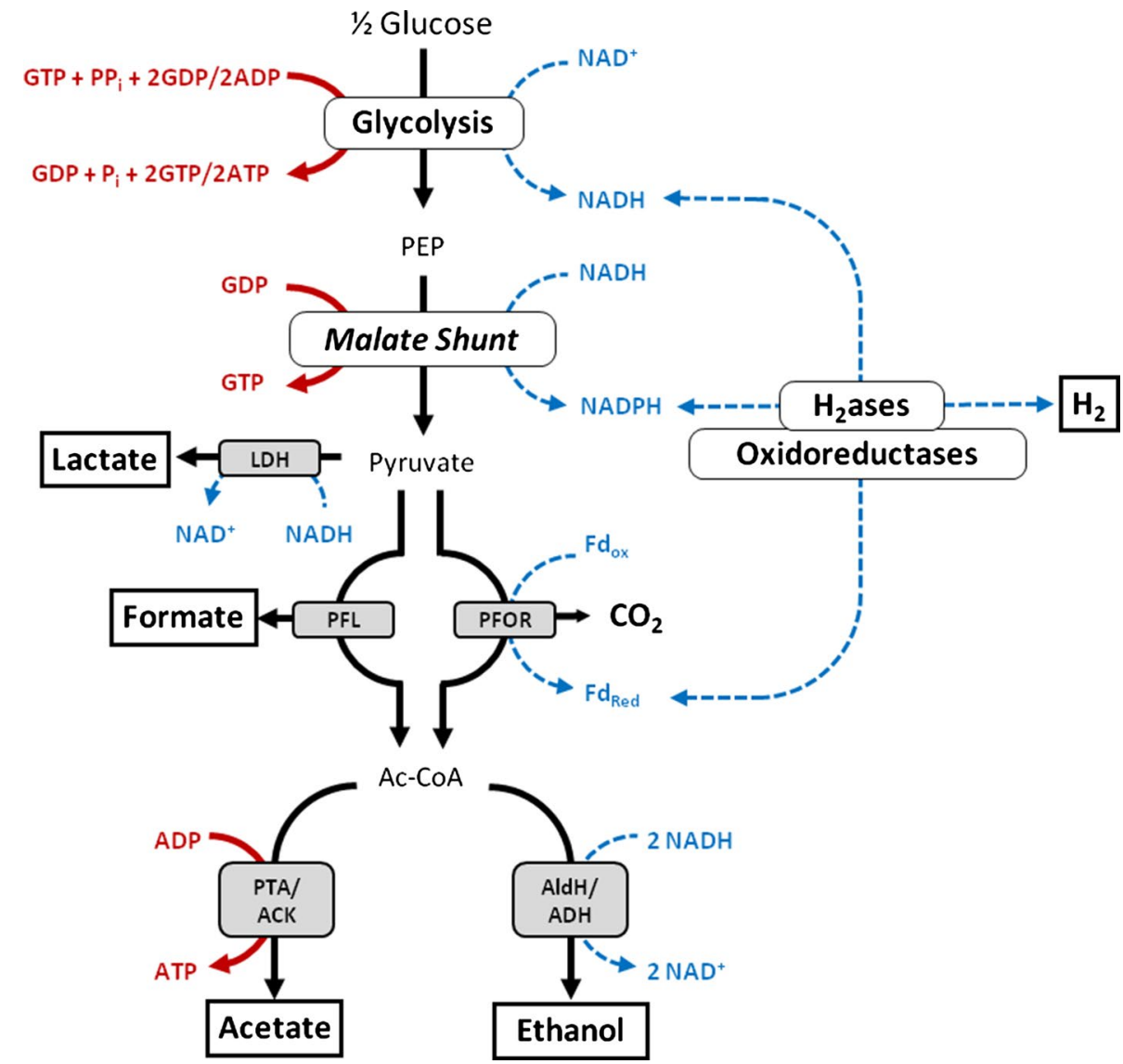

a dedicated step for cellulase production [23-25]. While a number of organisms are capable of cellulase-mediated cellulose hydrolysis and subsequent fermentation to ethanol, none have been yet identified or developed that can produce ethanol at high yields and titer required for commercial production.

Clostridium thermocellum is a promising candidate for ethanol production via CBP given its inherent ability to rapidly solubilize cellulose and ferment the hydrolysis products to biofuels (i.e., ethanol and $\mathrm{H}_{2}$ ) $[13,21,22]$. However, branched metabolic pathways divert carbon and/ or electrons away from ethanol towards undesired fermentation products including formate, $\mathrm{H}_{2}$, lactate, and acetate (Fig. 1). Many of these pathways have been elucidated through enzymology [15, 20, 28, 32, 37, 38, 44], transcriptomics [5, 29, 42, 43], proteomics [30, 31, 34], and genetics [1, 3, 7, 39]. More recent studies have demonstrated that carbon and electron flux are also diverted towards secreted amino acids [8, 14, 41] and other compounds including pyruvate, malate, fumarate, isobutanol, and butanediol, [12], further limiting ethanol yields.

While a number of studies have demonstrated that manipulation of fermentation conditions can influence product yields $[5,16,33]$, engineering of a $C$. thermocellum strain capable of high-yield ethanol production will require the elimination of the pathways involved in production of competing fermentation products. Recent advances related to genetic tools applicable to $C$. thermocellum, including electrotransformation protocols [27, 40], positive and negative selection systems used to select for gene deletions [1, 39], and improvement of transformation efficiencies through elimination of Dcm methylation of plasmid DNA [9] have allowed for genetic engineering of $C$. thermocellum. These tools have been used to begin engineering C. thermocellum to increase ethanol yield. Previous deletion of genes involved in many competing pathways have included phosphotransacetylase (pta; [1, 39]), lactate dehydrogenase $(l d h ;[1,2])$, malate dehydrogenase ( $m d h ;[7])$, malic enzyme (malE; [7]), Ech-type ferredoxin-dependent hydrogenase (ech; [3]), and a [FeFe] hydrogenase maturation factor to inactivate $[\mathrm{FeFe}]$ hydrogenases (hydG; [3]). Further, to increase NADH availability for bifunctional aldehyde/alcohol dehydrogenase ( $a d h E)$, heterologous expression of pyruvate kinase was used to bypass the 'malate shunt' to reduce transhydrogenation that generates NADPH from using electrons from NADH [7], and expression of a mutant AdhE allele was also shown to increase ethanol tolerance in C. thermocellum [4].

While most pathways to products other than ethanol have been deleted in $C$. thermocellum, this is not the case 
for formate synthesis. Production of reduced compounds such as formate limits the electrons available for ethanol production; therefore, identifying and deleting the gene(s) involved in formate synthesis are important next steps in the engineering of $C$. thermocellum for high-yield ethanol production for CBP.

In this study, we simultaneously deleted pyruvate:formate lyase ( $p f B$; Clo1313_1717) and Pfl-activating enzyme ( $p f A$; Clo1313_1716) in C. thermocellum in an attempt to increase electron flux towards ethanol and to understand how this mutation impacts growth, end-product synthesis, and amino acid secretion on rich and defined medium.

\section{Materials and methods}

\section{Strains, culture conditions, and reagents}

Saccharomyces cerevisiae InvSc1 (uracil auxotroph; Life Technologies, Grand Island, NY), used for yeast gap repair cloning, was maintained on YPD medium and grown on SD-ura medium (Sunrise Science Products, San Diego, CA) when selecting for presence of $\mathrm{URA}^{+}$plasmids.

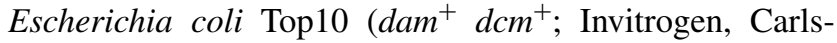
bad, CA) and BL21 (dam ${ }^{+} \mathrm{dcm}^{-}$; New England Biolabs, Ipswich, MA) strains were used for plasmid construction and propagated aerobically on LB medium supplemented with $12 \mu \mathrm{g} / \mathrm{ml}$ chloramphenicol as required for plasmid maintenance. Clostridium thermocellum strains were derived from strain DSM 1313 (Deutsche Sammlung von Microorganismen und Zellkulturen, Braunshwieg, Germany) and were routinely grown anaerobically at $55{ }^{\circ} \mathrm{C}$, unless otherwise noted, in a Coy anaerobic chamber (Coy Laboratory Products, Grass Lake, MI) on $5 \mathrm{~g} / \mathrm{l}$ cellobiose in modified DSM 122 complex medium supplemented with $50 \mathrm{mM}$ MOPS and $10 \mathrm{mM}$ sodium citrate [39] (referred to as CTFUD). Medium was made anaerobic via autoclaving to remove $\mathrm{O}_{2}$ from solution, followed by immediate transfer to the anaerobic chamber to maintain anaerobicity and was supplemented with $10 \mu \mathrm{g} / \mathrm{ml}$ thiamphenicol, $50 \mu \mathrm{g} /$ $\mathrm{ml}$ 5-fluoro-2'-deoxyuridine, or $500 \mu \mathrm{g} / \mathrm{ml}$ 8-azahypoxanthine (Tokyo Chemical Industry, Co., Tokyo, Japan) during $C$. thermocellum strain construction when appropriate. C. thermocellum strains were grown in either CTFUD or a modified version of chemically defined Medium for Thermophilic Clostridia (MTC; [36]) in which (i) urea concentrations were reduced and medium was supplemented with trace elements and additional vitamins as outlined by [2] and (ii) all stock solutions were filter sterilized rather than autoclaved. This modified version of MTC is now referred to as ' $\mathrm{MTC}_{5}$ '. Tubes containing either CTFUD or $\mathrm{MTC}_{5}$ were made anaerobic by degassing/gassing $(2: 1 \mathrm{~min})$ ten times with $100 \% \mathrm{~N}_{2}$. Final pressure of tubes was equilibrated to 5 psi over ambient pressure. All chemicals were reagent grade and obtained from Sigma-Aldrich (St. Loius, MO) or Fisher Scientific (Waltham, MA) unless otherwise specified.

\section{Plasmid and strain construction}

All plasmids used in this study were constructed using yeast gap repair cloning as outlined by Shanks et al. [35]. Plasmid isolation and purification were performed using QIAprep spin miniprep kits (QIAGEN, Germantown, MD). C. thermocellum $\Delta h p t$ [1] was transformed via electroporation as previously described $[9,27]$. The pflAB genes were deleted according to the protocol developed in Argyros et al. [1] and detailed in Olson et al. [27] using plasmid pAMG281 (Accession number KP864661; Online Resource 1). Gene deletion was confirmed using primer sets $\mathrm{P}_{\mathrm{F}} \quad\left(5^{\prime}\right.$-GAAATTATACTCCTTATGAA GGCGA-3') and $\mathrm{P}_{\mathrm{R}}$ (5'-TCTGTTCCTTGACTGCTG CAA-3'); P2 ${ }_{\mathrm{F}}$ (5'-CGGAGCCCAACCTTACAGTAC-3') and $\mathrm{P} 2_{\mathrm{R}}$ (5'-TATGGAAAGGGTCGGAGTGG-3'); and $\mathrm{P}_{\mathrm{F}}\left(5^{\prime}\right.$-ATACTTGATTATTATGAGCGCGG-3') and $\mathrm{P} 3_{\mathrm{R}}$ (5'-ATTCTCCTGGTTAAGCCTTGTAA- $3^{\prime}$ ) as described below.

\section{Fermentation conditions}

Fermentation experiments were carried out in sealed Balch tubes (27 ml; Belco Glass Inc., Vineland, NJ) containing either CTFUD or $\mathrm{MTC}_{5}$ as described above. C. thermocellum inoculum was subcultured a minimum of three times using a $2 \%(\mathrm{v} / \mathrm{v})$ inoculum on corresponding medium to prevent carry over. Fermentations were performed on $4.5 \mathrm{~g} / \mathrm{l}$ cellobiose at $55{ }^{\circ} \mathrm{C}$ until all substrate was consumed. Final fermentation products were measured following complete cellobiose utilization $(<0.25 \mathrm{mM}$ remaining). Fermentations were performed a minimum of two times with three independent biological replicates each time. For growth curves, cells were grown in $650 \mu \mathrm{l}$ total volume and growth was monitored spectrophotometrically at $\mathrm{OD}_{600}$ in an Eon Microplate Spectrophotometer (BioTek Instruments Inc., Winooski, VT) situated in a Coy anaerobic chamber.

\section{Analytical methods}

Substrate (cellobiose), the cellobiose hydrolysis product glucose, and fermentation products (pyruvate, lactate, acetate, formate, ethanol) were analyzed using a Breeze High Performance Liquid Chromatography system (Waters, Milford, MA) using an Aminex-HPX-87H column (BioRad, Hercules, CA) with a $5 \mathrm{mM}$ sulfuric acid mobile phase. $\mathrm{H}_{2}$ was measured using an Agilent Technologies 
6850 Series II Gas Chromatograph (Agilent Technologies, Santa Clara, CA) using a thermoconductivity detector set at $190{ }^{\circ} \mathrm{C}$ with an $\mathrm{N}_{2}$ reference flow and a Carbonex 1010 PLOT (30.0 m $\times 530 \mu \mathrm{m}$ I.D.; model Supelco 25467) column. Secreted amino acids were measured using an Aracus Amino Acid Analyzer (membraPure, Berlin, Germany) using a T111 Li-cation exchange column with eluents supplied by the manufacturer. Ninhydrin-derived amino acids were measured photometrically at $570 \mathrm{~nm}$ with the exception of proline, which was measured at $440 \mathrm{~nm}$. Final $\mathrm{pH}$ was measured using an Accument AB15 Basic pH meter (Fisher Scientific; Pittsburg, PA).

\section{Calculations}

$\mathrm{CO}_{2}$ produced was calculated based on the expected ratio of $\mathrm{C}_{1}: \mathrm{C}_{2}$ compounds and the fact that valine biosynthesis also liberates $\mathrm{CO}_{2}$, whereby $\mathrm{CO}_{2}=[($ ethanol + acetate $)-($ formate)] + valine. Ratios of oxidized to reduced fermentation products $(\mathrm{O} / \mathrm{R})$ were calculated using reduction values of each fermentation product, calculated as the number of oxygen atoms less one-half the number of hydrogens in each compound [26]. Carbon bound electron equivalents were calculated as described by Harris et al. [10] and were used to determine electron recovery.

\section{Results}

\section{Deletion of $C$. thermocellum $p f$ eliminates formate and decreases acetate production}

The genes encoding pyruvate-formate lyase ( $p f l B)$ and Pflactivating enzyme ( $p f A$ ) were deleted in $C$. thermocellum $\Delta h p t$, confirmed using three primer sets as described in Online Resource 2, and is hereafter referred to as $C$. thermocellum $\Delta p f$.

C. thermocellum $\Delta h p t$ and $\Delta p f l$ were grown on $4.5 \mathrm{~g} / \mathrm{l}$ $(13.1 \mathrm{mM})$ cellobiose in complex (CTFUD) medium or defined medium $\left(\mathrm{MTC}_{5}\right)$. Final fermentation products were measured following nearly complete fermentation of cellobiose $(<0.25 \mathrm{mM}$ remaining). Deletion of $p f$ eliminated formate production and decreased acetate production by $\sim 50 \%$, regardless of medium used (Fig. 2). However, there were significant medium-dependent differences in other final fermentation products. On CTFUD, no differences were observed in final $\mathrm{H}_{2}$, ethanol, or lactate concentrations in $\Delta p f l$ cultures when compared to $\Delta h p t$. However, when $C$. thermocellum was grown on $\mathrm{MTC}_{5}, \Delta p f l$ cultures produced 1.4-fold more $\mathrm{H}_{2}$ and 9.3-fold more lactate compared to the wild type along with comparable concentrations of ethanol. Notably, $\Delta h p t$ cultures grown on CTFUD produced 15-fold more lactate than on $\mathrm{MTC}_{5}$. Residual
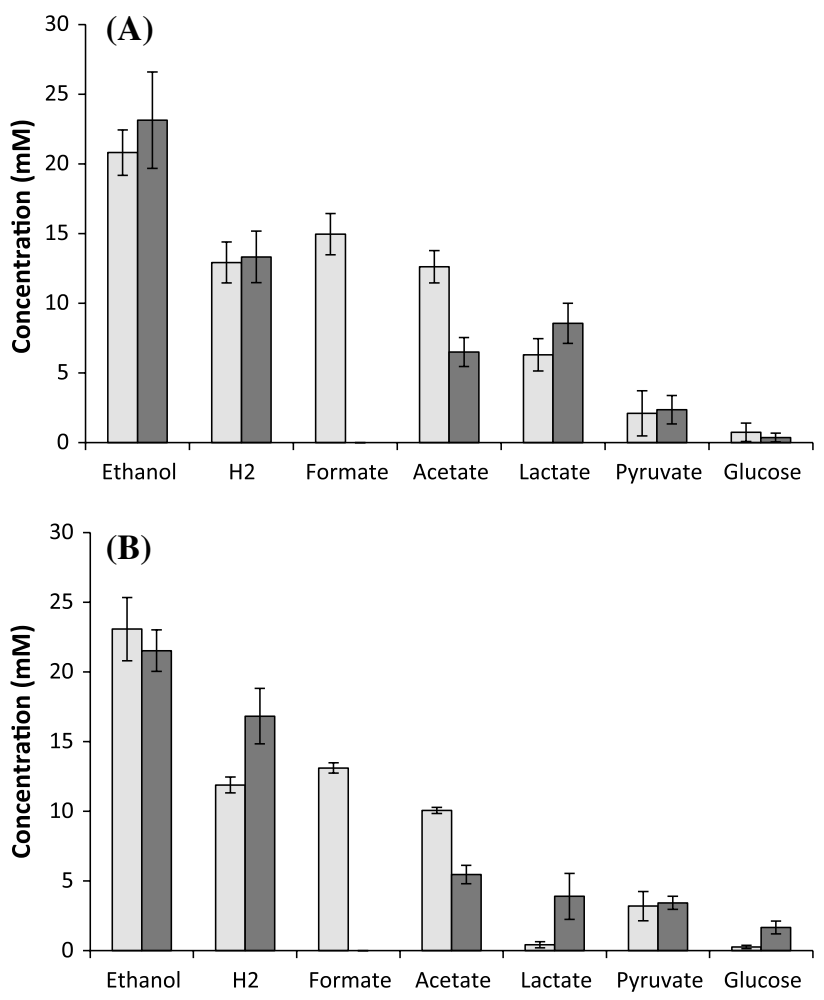

Fig. 2 Final fermentation products of $C$. thermocellum strains on a complex medium (CTFUD) and $\mathbf{b}$ defined medium $\left(\mathrm{MTC}_{5}\right)$. All fermentation products were measured upon completion of cellobiose utilization. Light grey bars, $\Delta$ hpt; Dark grey bars, $\Delta p f$

glucose was detected at the end of growth in all cultures and ranged from $0.3 \mathrm{mM}$ in $\Delta h p t$ cultures grown on $\mathrm{MTC}_{5}$ to $1.7 \mathrm{mM}$ in $\Delta p f l$ cultures grown on $\mathrm{MTC}_{5}$ (Fig. 2, Online Resource 3). Secreted pyruvate was also detected on CTFUD (2.1 and $2.4 \mathrm{mM}$ ) and $\mathrm{MTC}_{5}(3.2$ and $3.4 \mathrm{mM})$ in $\Delta h p t$ and $\Delta p f l$ cultures, respectively (Fig. 2, Online Resource 3).

Given that the addition of sodium bicarbonate to $\mathrm{MTC}_{5}$ rendered accurate measurements of $\mathrm{CO}_{2}$ infeasible, $\mathrm{CO}_{2}$ was calculated based on detected fermentation products (Table 1). As expected, deletion of $p f l$ resulted in an increase in calculated $\mathrm{CO}_{2}$ production of 1.6- and 1.3-fold on CTFUD and $\mathrm{MTC}_{5}$, respectively. Oxidation/reduction balances $(\mathrm{O} / \mathrm{R})$ based on formate, ethanol, $\mathrm{H}_{2}$, and calculated $\mathrm{CO}_{2}$ were $\sim 1$, in support of the validity of our carbon accounting assumptions.

\section{Select amino acids are utilized by $C$. thermocellum}

Changes in medium amino acid concentrations at the end of growth are depicted in Fig. 3. Valine and alanine were secreted at the highest levels, regardless of medium or strain used. For CTFUD, which contains yeast extract (Online Resource 3), glutamate and leucine were consumed 
Table 1 Final product yields and fermentation balances of $\Delta h p t$ and $\Delta p f l$ on CTFUD and MTC

\begin{tabular}{|c|c|c|c|c|c|c|c|c|c|c|c|c|c|}
\hline & \multicolumn{6}{|c|}{ Product yields ( $\mathrm{mol} \mathrm{mol-hexose} \mathrm{consumed}{ }^{-1}$ ) } & \multirow[t]{2}{*}{$\mathrm{O} / \mathrm{R}_{\text {balance }}{ }^{\mathrm{b}}$} & \multicolumn{3}{|c|}{$\mathrm{C}$ recovery $(\%)^{\mathrm{c}}$} & \multicolumn{3}{|c|}{$\mathrm{e}^{-}$recovery $(\%)^{\mathrm{c}}$} \\
\hline & Ethanol & $\mathrm{H}_{2}$ & Formate & Acetate & Lactate & $\mathrm{CO}_{2}^{\mathrm{a}}$ & & FPs & AAs & Total & FPs & AAs & Total \\
\hline \multicolumn{14}{|c|}{ CTFUD } \\
\hline$\Delta h p t$ & 0.77 & 0.48 & 0.56 & 0.47 & 0.23 & 0.74 & 1.05 & 84.5 & 1.6 & 86.2 & 83.7 & 1.1 & 85.9 \\
\hline$\Delta p f l$ & 0.87 & 0.50 & 0.00 & 0.25 & 0.32 & 1.19 & 1.09 & 79.3 & -0.7 & 78.5 & 77.5 & -0.1 & 77.5 \\
\hline \multicolumn{14}{|l|}{ MTC } \\
\hline$\Delta h p t$ & 0.87 & 0.45 & 0.50 & 0.38 & 0.02 & 0.83 & 1.04 & 72.5 & 7.7 & 80.2 & 71.8 & 9.0 & 80.8 \\
\hline$\Delta p f l$ & 0.81 & 0.63 & 0.00 & 0.21 & 0.15 & 1.07 & 1.01 & 73.4 & 3.4 & 76.8 & 73.3 & 4.2 & 77.5 \\
\hline
\end{tabular}

Carbon recoveries of fermentation products (FPs), amino acids (AAs), and total secreted products are provided

a $\mathrm{CO}_{2}$ was calculated using $\mathrm{C}_{1}: \mathrm{C}_{2}$ ratio using ethanol, acetate and formate concentrations

b $\mathrm{O} / \mathrm{R}$ balance was determined using formate, calculated $\mathrm{CO}_{2}$ (from $\mathrm{C} 1: \mathrm{C} 2$ ratio), $\mathrm{H}_{2}$, and ethanol

${ }^{c}$ Carbon and electron recoveries were calculated based on all substrates (cellobiose and glucose) and end-products (ethanol, $\mathrm{H}_{2}$, formate, acetate, lactate, pyruvate) detected, calculated $\mathrm{CO}_{2}$ (from $\mathrm{C}_{1}: \mathrm{C}_{2}$ ratio), and secreted amino acids, but exclude biomass measurements

Fig. 3 Final secreted amino acid concentrations of $C$. thermocellum strains on a complex medium (CTFUD) and $\mathbf{b}$ defined medium $\left(\mathrm{MTC}_{5}\right)$. All fermentation products were measured upon completion of cellobiose utilization. Light grey bars $\Delta$ hpt; Dark grey bars $\Delta p f$; (Cys)2, cystine. Negative values indicate net consumption of the given amino acid. Error bars represent one standard deviation from the mean
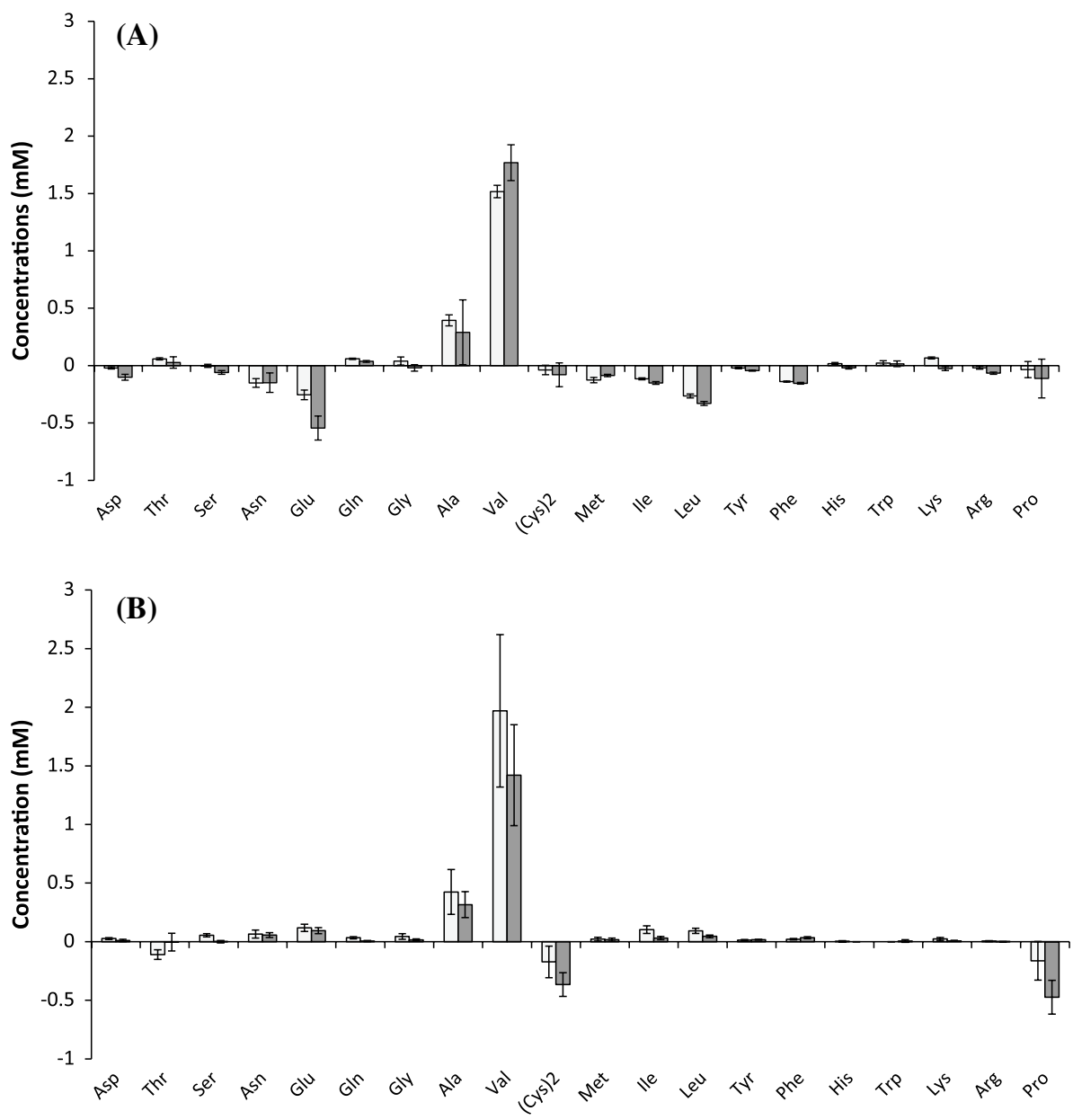

by both $\Delta h p t$ ( 0.4 and $0.3 \mathrm{mM}$, respectively) and $\Delta p f$ (0.7 and $0.3 \mathrm{mM}$, respectively). All other amino acids were consumed or produced at concentrations less than $0.15 \mathrm{mM}$ (Fig. 3a) on CTFUD. Secreted valine concentrations were 1.5 and $1.8 \mathrm{mM}$, while secreted alanine concentrations were 0.4 and $0.3 \mathrm{mM}$, respectively, for $\Delta h p t$ and
$\Delta p f l$ on CTFUD. While $\mathrm{MTC}_{5}$ was not supplemented with amino acids other than cysteine (used as a reducing agent), $1.4 \mathrm{mM}$ proline and $0.3 \mathrm{mM}$ threonine were detected in uninoculated $\mathrm{MTC}_{5}$. Consumption of both cysteine and proline was observed by both strains in MTC, albeit consumption of each was greater by $\Delta p f l$ (Fig. 3b). While final 

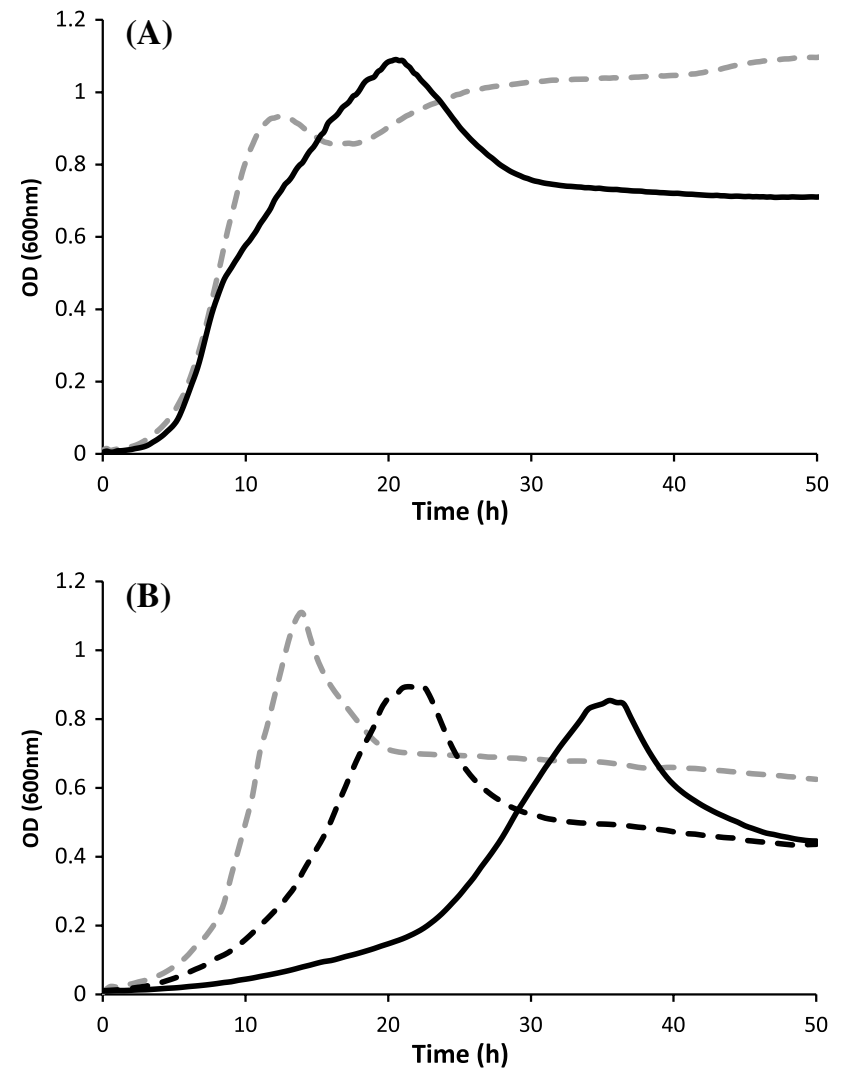

Fig. 4 Growth profiles of parent and mutant strains on a complex medium (CTFUD) and $\mathbf{b}$ defined medium $\left(\mathrm{MTC}_{5}\right)$. Dashed grey line $\Delta h p t ;$ solid black line $\Delta p f$; dashed black line $\Delta p f$ supplemented with $2 \mathrm{mM}$ formate

concentrations of valine were 2.0 and $1.4 \mathrm{mM}$ for $\Delta h p t$ and $\Delta p f l$, respectively, and those for alanine were 0.4 and $0.3 \mathrm{mM}$ for $\Delta h p t$ and $\Delta p f$, respectively, concentrations of all other secreted amino acids were less than $0.15 \mathrm{mM}$.

Total carbon and electron recoveries in fermentation products were similar between strains under each growth condition and ranged from 77 to $86 \%$ (Table 1). Net production of amino acids accounted for ca. $2 \%$ of final carbon and electron balances in CTFUD-grown $\Delta h p t$ supernatants, whereas no net difference in amino acids was observed in CTFUD-grown $\Delta p f$ supernatants. On $\mathrm{MTC}_{5}$, however, total secreted amino acids accounted for ca. $8 \%$ of carbon and electron recoveries in $\Delta h p t$ supernatants, and ca. $4 \%$ in $\Delta p f l$ supernatants (Table 1, Online Resource 3).

\section{Growth rate is altered in $C$. thermocellum $\Delta p f$}

To further examine the effect of $p f l$ deletion in $C$. thermocellum, we examined growth of the parent strain and the $\Delta p f$ mutant on cellobiose in CTFUD and MTC $_{5}$. Growth of the parent strain and $\Delta p f l$ were similar on complex CTFUD for the first $7 \mathrm{~h}$, with an exponential-phase generation time of $1.3 \mathrm{~h}( \pm 0.1)$ and $1.2 \mathrm{~h}( \pm 0.2)$, respectively (Fig. 4a). At an optical density of ca. $0.5, \Delta p f l$ began to grow slower $[9.3 \mathrm{~h}( \pm 2.7)$ doubling time] than the parent strain for an additional $10 \mathrm{~h}$ until growth peaked at an $\mathrm{OD}_{600}$ of 1.1. The final $\mathrm{pH}$ of $\Delta h p t$ and $\Delta p f l$ cultures was 5.9 and 6.3 , respectively.

On defined medium $\mathrm{MTC}_{5}$, both $\Delta h p t$ and $\Delta p f$ exhibited a typical-shaped growth curve (Fig. 4b). While $\Delta h p t$ grew to the same maximum $\mathrm{OD}_{600}(1.1)$ on defined medium when compared to CTFUD, its exponential-phase generation time increased to $2.0 \mathrm{~h}( \pm 0.4)$. Deletion of $p f$ almost tripled the generation time to $6.0 \mathrm{~h}( \pm 1.6)$ and marginally decreased maximum $\mathrm{OD}_{600}$ to 0.9 when compared to the parent strain. The final $\mathrm{pH}$ of $\Delta h p t$ and $\Delta p f$ cultures in $\mathrm{MTC}_{5}$ medium was 7.0 and 6.7, respectively. In Staphylococcus aureus, growth of a $\mathrm{pfl}$ mutant was improved by addition of $2 \mathrm{mM}$ formate [17]. Therefore, we supplemented $\mathrm{MTC}_{5}$ with $2 \mathrm{mM}$ formate, which decreased the generation time of $\Delta p f l$ to $3.4( \pm 0.5)$ but had negligible impact on maximum $\mathrm{OD}_{600}$.

\section{Discussion}

Improvement of $C$. thermocellum ethanol yields may be achieved through elimination of branched fermentation pathways that divert carbon and electron flux away from ethanol. Previous reports have demonstrated that deletion of genes responsible for acetate [1, 39], lactate [1, 2], and $\mathrm{H}_{2}$ synthesis [3] can increase ethanol yields. Production of formate, which has also been shown to be a major fermentation product of $C$. thermocellum [37], can also reduce the amount of electrons that are available for ethanol production [31]. Deletion of $p f l$ completely eliminated formate production, demonstrating that it encodes the only functional pathway to formate synthesis in C. thermocellum DSM 1313.

The amount of formate produced by $C$. thermocellum varied considerably in previous studies and comparisons are complicated by differences in medium composition, $\mathrm{pH}$ control, headspace gas accumulation, carbon loading, and batch vs. continuous fermentation. Typical formate yields in C. thermocellum DSM 1313 wild type ranges from 0.05 to 0.15 mol mol-hexose ${ }^{-1}[11,41,44]$ but can be as high as

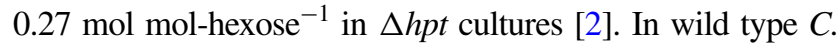
thermocellum strain ATCC 27405, reported formate yields range from 0.07 to 0.48 [5, 8, 31, 32]. Here we observed formate yields of 0.57 and $0.50 \mathrm{~mol} \mathrm{mol- \textrm {glc } ^ { - 1 }}$ for wild-type strains on CTFUD and $\mathrm{MTC}_{5}$, respectively, both of which were on the higher end of the spectrum when compared to previous results. This variability in medium and/or strain-dependent yields could prove problematic for applied processes; however, by deleting $p f l$, this variable flux to formate is eliminated during $C$. thermocellum cellulose fermentations. 
In the absence of $p f$, conversion of pyruvate to acetyl$\mathrm{CoA}$ is only catalyzed by pyruvate:ferredoxin $(\mathrm{Fd})$ oxidoreductase (PFOR), which generates $\mathrm{CO}_{2}$ and reduced $\mathrm{Fd}$ (Fig. 1). Calculated final $\mathrm{CO}_{2}$ concentrations were higher in $\Delta p f$ when compared to $\Delta h p t$ by 1.6 and 1.4 -fold on CTFUD and $\mathrm{MTC}_{5}$, respectively, suggesting that carbon and electron flux through PFOR is increased. This is in agreement with previous studies in which PFL activity in $C$. thermocellum ATCC 27405 was inhibited using hypophosphite [31]. Despite the increase of additional reducing equivalents produced in the form of reduced $\mathrm{Fd}$, changes in ethanol production were minimal, whereas acetate production decreased by ca. $50 \%$, regardless of medium used. Consequently, the increase in calculated $\mathrm{CO}_{2}$ production was only 76 and $49 \%$ of the decrease in formate production on CTFUD and $\mathrm{MTC}_{5}$, respectively, demonstrating that increased flux through PFOR does not fully compensate for loss of $p f l$, and reduced overall flux from pyruvate to acetylCoA may lead to a build-up of intermediates upstream of acetyl-CoA that are diverted away from acetate and ethanol.

On $\mathrm{MTC}_{5}$, deletion of $p f$ increased carbon and electron flux towards lactate and $\mathrm{H}_{2}$ and decreased carbon flux towards acetate. Given that lactate dehydrogenase requires fructose-1,6-bisphosphate $(\mathrm{FbP})$ as an allosteric activator [28, 32], it may be that higher lactate production in the $\Delta p f l$ strain is a result of $\mathrm{FbP}$ accumulation due to restrictions on the rate of glycolytic flux when conversion of pyruvate to acetyl-CoA is catalyzed by PFOR without PFL. Surprisingly, ethanol production did not increase in the $\Delta p f l$ strain, consistent with potential limitation by acetyl-CoA or NADH availability. On CTFUD, only a marginal increase in lactate and ethanol was observed, while $\mathrm{H}_{2}$ production did not change, suggesting that carbon and electron flux is diverted elsewhere. The two most abundant secreted amino acids, valine and alanine, are derived from pyruvate; therefore, one might hypothesize that the reduced flux from pyruvate to acetyl-CoA might increase flux to secreted amino acids. However, amino acid secretion in fact decreased in $\Delta p f l$ cultures when compared to $\Delta h p t$, regardless of medium used, suggesting that carbon and electron flux is diverted elsewhere.

While deletion of $p f l$ eliminated formate production, growth of the $\Delta p f l$ strain was hindered, especially in minimal medium. On $\mathrm{MTC}_{5}$ the exponential phase growth rate of the $\Delta p f l$ strain was reduced to $40 \%$ of that of the parent strain, and final $\mathrm{OD}_{600}$ decreased by $21 \%$. Given that the $\Delta p f l$ strain produced $\sim 50 \%$ less acetate, a decrease in ATP availability could partially explain lower final growth yields. Interestingly, while deletion of $p f l$ could potentially decrease metabolic flux upstream of acetyl-CoA and, in turn, decrease growth rate, exponential phase growth rate was restored to $80 \%$ of that of the parent strain when $\mathrm{MTC}_{5}$ was supplemented with $2 \mathrm{mM}$ formate. Similar restoration of growth rate was observed with formate supplementation for a Staphylococcus aureus $\Delta p f l$ mutant [17]. Formate is commonly used for formyl-tetrahydrofolate (THF) synthesis via formate-THF ligase (Clo1313_0030). Formyl-THF in turn acts as a key donor of formyl groups required for purine and formylmethionine synthesis, as well as methyl group donor for synthesis of methionine and S-adenosylmethionine (Fig. 5). Thus, we hypothesize that elimination of formate production in $C$. thermocellum has an adverse impact on $\mathrm{C}_{1}$ metabolism, causing a growth defect. Despite this, the $\Delta p f l$ strain is able to grow in minimal medium without formate supplementation, albeit poorly, indicating that $C$. thermocellum has an alternate route for synthesizing formyl- and methyl-THF. In the absence of formate supplementation or synthesis via PFL, we postulate that serine may be a precursor for formyl-THF synthesis (Fig. 5). While genomic analysis reveals the presence of all enzymes required for formyl-THF synthesis from serine in $C$. thermocellum, the pathway for serine biosynthesis is unclear. Possible routes could include phosphoserine phosphatases (PSPH) or serine-pyruvate transaminases (SPT), but none are annotated in $C$. thermocellum. One possibility is that PSPH activity is present in C. thermocellum but is encoded by an uncharacterized phosphatase. Alternatively, the annotated alanine-glyoxylate transaminase (AGAT) may have SPT activity, synthesizing serine from hydroxypyruvate. Indeed, other studies have demonstrated that the Arabidopsis AGAT can have both AGAT and SPT activities [18, 19]. Regardless of the pathway used to make formyl- and methyl-THF in $\Delta p f l$, this pathway is clearly less efficient than the native pathway utilizing formate. Future improvement in formyl- and methyl-THF synthesis in the absence of $p f$ will likely improve the growth rate of these strains.

\section{Conclusion}

In this study, we have eliminated formate production in $C$. thermocellum by deleting genes encoding PFL and PFLactivating enzyme. Redirecting metabolic flux away from PFL towards PFOR could have generated additional electrons available for ethanol production. Although ethanol yields did not increase, other fermentation products that act as electron sinks (i.e., $\mathrm{H}_{2}$ and lactate) did increase. Thus, future strategies to improve ethanol yields may involve deletion of lactate and $\mathrm{H}_{2}$-formation pathways in conjunction with deletion of PFL to further limit electron flux towards ethanol. Here we demonstrate that formate availability is important in maintaining growth rate in $C$. thermocellum and propose that it acts as a precursor for methyland formyl-THF biosynthesis, and ultimately methionine, purine and formylmethionine synthesis. Thus, future improvement in formyl-THF synthesis in the absence of $p f$ 


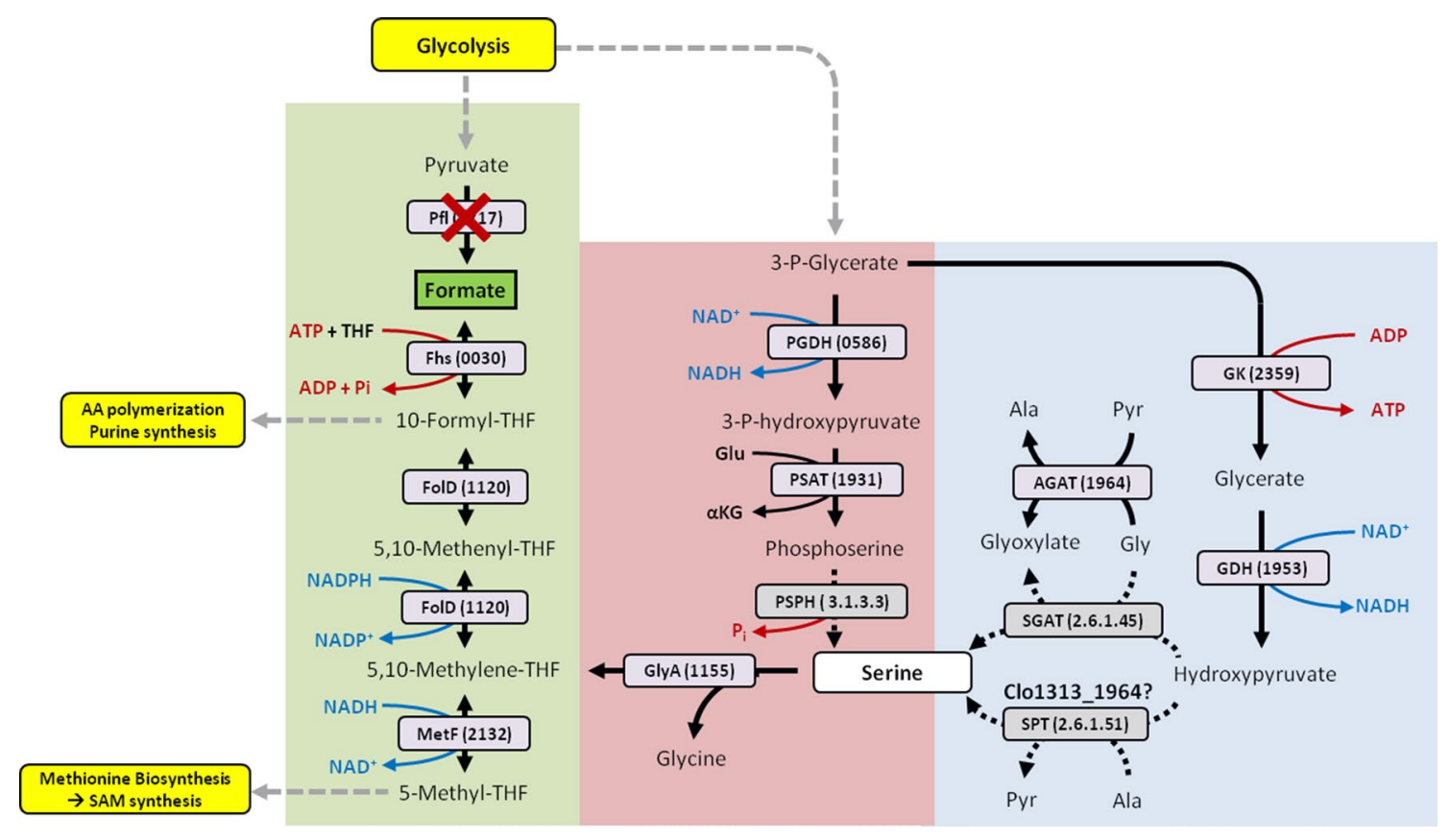

Fig. 5 Proposed formyl-THF synthesis pathway in the presence and absence of formate. Formyl-THF may be synthesized directly from formate via formate-THF ligase (Fhs). In the absence of formate, we propose that serine is a precursor for formyl-THF synthesis. While no annotated phosphoserine phosphatase (PSPH) or serine-pyruvate aminotransferase (SPT) is annotated in C.thermocellum, alanineglyoxylate transaminase (AGAT) may function as a SPT. Annotated gene numbers (Clo1313) are indicated in brackets. Enzyme

will likely improve the growth rate of these strains, which will be required to reduce energy inputs and costs of largescale fermentations.

Acknowledgments This work was supported by the BioEnergy Science Center, U.S. DOE Bioenergy Research Center supported by the Office of Biological and Environmental Research in the DOE Office of Science. Oak Ridge National Laboratory is managed by UT-Battelle, LLC, for the U.S. DOE under contract DE-AC05-00OR22725. The funders had no role in study design, data collection and analysis, decision to publish, or preparation of the manuscript.

\section{Compliance with ethical standards}

Conflict of interest Lee Lynd is an employee of Mascoma Corporation, which has a financial interest in C. thermocellum

Ethical approval This article does not contain any studies with human participants or animals performed by any of the authors.

Open Access This article is distributed under the terms of the Creative Commons Attribution 4.0 International License (http://creativecommons.org/licenses/by/4.0/), which permits unrestricted use, distribution, and reproduction in any medium, provided you give appropriate credit to the original author(s) and the source, provide a link to the Creative Commons license, and indicate if changes were made.
E.C. numbers are provided for enzymes which are not annotated in C. thermocellum. THF tetrahydrofolate; $G K$ glycerate kinase; $G D H$ glycerate dehydrogenase; $P G D H$ 3-phosphoglycerate dehydrogenase; PSAT phosphoserine aminotransferase); SGAT serine-glyoxylate aminotransferase; GlyA serine hydroxymethyltransferase; $M e t F$ methylene-THF reductase; FolD bifunctional methylene-THF dehydrogenase/methylene-THF cyclohydrolase; $P f$ pyruvate:formate lyase

\section{References}

1. Argyros DA, Tripathi SA, Barrett TF, Rogers SR, Feinberg LF, Olson DG, Foden JM, Miller BB, Lynd LR, Hogsett DA, Caiazza NC (2011) High ethanol titers from cellulose using metabolically engineered thermophilic, anaerobic microbes. Appl Environ Microbiol 77:8288-8294. doi:10.1128/aem.00646-11

2. Biswas R, Prabhu S, Lynd LR, Guss AM (2014) Increase in Ethanol Yield via Elimination of Lactate Production in an Ethanol-Tolerant Mutant of Clostridium thermocellum. PLoS One 9:e86389. doi:10.1371/journal.pone.0086389

3. Biswas R, Zheng T, Olson DG, Lynd LR, Guss AM (2015) Elimination of hydrogenase active site assembly blocks $\mathrm{H} 2$ production and increases ethanol yield in Clostridium thermocellum. Biotechnol Biofuels 8. doi:10.1186/s13068-015-0204-4

4. Brown SD, Guss AM, Karpinets TV, Parks JM, Smolin N, Yang S, Land ML, Klingeman DM, Bhandiwad A, Rodriguez M Jr, Raman B, Shao X, Mielenz JR, Smith JC, Keller M, Lynd LR (2011) Mutant alcohol dehydrogenase leads to improved ethanol tolerance in Clostridium thermocellum. Proc Natl Acad Sci U S A 108:13752-13757. doi:10.1073/pnas.1102444108

5. Carere CR, Rydzak T, Cicek N, Levin DB, Sparling R (2014) Role of transcription and enzyme activities in redistribution of carbon and electron flux in response to $\mathrm{N}(2)$ and $\mathrm{H}(2)$ sparging of open-batch cultures of Clostridium thermocellum ATCC 27405. Appl Microbiol Biotechnol 98:2829-2840. doi:10.1007/s00253-013-5500-y

6. Chu S, Goldemburg J (2007) Lighting the way: toward a sustainable energy future. InterAcademy Council, Amsterdam 
7. Deng Y, Olson DG, Zhou J, Herring CD, Joe Shaw A, Lynd LR (2013) Redirecting carbon flux through exogenous pyruvate kinase to achieve high ethanol yields in Clostridium thermocellum. Metab Eng 15:151-158. doi:10.1016/j. ymben.2012.11.006

8. Ellis LD, Holwerda EK, Hogsett D, Rogers S, Shao X, Tschaplinski T, Thorne P, Lynd LR (2012) Closing the carbon balance for fermentation by Clostridium thermocellum (ATCC 27405). Bioresour Technol 103:293-299. doi:10.1016/j. biortech.2011.09.128

9. Guss AM, Olson DG, Caiazza NC, Lynd LR (2012) Dcm methylation is detrimental to plasmid transformation in Clostridium thermocellum. Biotechnol Biofuels 5:30. doi:10.1186/1754-6834-5-30

10. Harris RF, Adams SS (1979) Determination of the carbon-bound electron composition of microbial cells and metabolites by dichromate oxidation. Appl Environ Microbiol 37:237-243

11. Holwerda EK, Hirst KD, Lynd LR (2012) A defined growth medium with very low background carbon for culturing Clostridium thermocellum. J Ind Microbiol Biotechnol 39:943-947. doi:10.1007/s10295-012-1091-3

12. Holwerda EK, Thorne PG, Olson DG, Amador-Noguez D, Engle NL, Tschaplinski TJ, van Dijken JP, Lynd LR (2014) The exometabolome of Clostridium thermocellum reveals overflow metabolism at high cellulose loading. Biotechnol Biofuels 7:155. doi:10.1186/s13068-014-0155-1

13. Islam R, Cicek N, Sparling R, Levin D (2009) Influence of initial cellulose concentration on the carbon flow distribution during batch fermentation by Clostridium thermocellum ATCC 27405. Appl Microbiol Biotechnol 82:141-148. doi:10.1007/ s00253-008-1763-0

14. Kridelbaugh DM, Nelson J, Engle NL, Tschaplinski TJ, Graham DE (2013) Nitrogen and sulfur requirements for Clostridium thermocellum and Caldicellulosiruptor bescii on cellulosic substrates in minimal nutrient media. Bioresour Technol 130:125135. doi:10.1016/j.biortech.2012.12.006

15. Lamed R, Zeikus JG (1980) Ethanol production by thermophilic bacteria: relationship between fermentation product yields of and catabolic enzyme activities in Clostridium thermocellum and Thermoanaerobium brockii. J Bacteriol 144:569-578

16. Lamed RJ, Lobos JH, Su TM (1988) Effects of stirring and hydrogen on fermentation products of Clostridium thermocellum. Appl Environ Microbiol 54:1216-1221

17. Leibig M, Liebeke M, Mader D, Lalk M, Peschel A, Gotz F (2011) Pyruvate formate lyase acts as a formate supplier for metabolic processes during anaerobiosis in Staphylococcus aureus. J Bacteriol 193:952-962. doi:10.1128/JB.01161-10

18. Liepman AH, Olsen LJ (2001) Peroxisomal alanine: glyoxylate aminotransferase (AGT1) is a photorespiratory enzyme with multiple substrates in Arabidopsis thaliana. Plant J 25:487-498

19. Liepman AH, Olsen LJ (2003) Alanine aminotransferase homologs catalyze the glutamate:glyoxylate aminotransferase reaction in peroxisomes of Arabidopsis. Plant Physiol 131:215227. doi:10.1104/pp.011460

20. Lin WR, Peng Y, Lew S, Lee CC, Hsu JJ, Hamel JF, Demain AL (1998) Purification and characterization of acetate kinase from Clostridium thermocellum. Tetrahedron 54:15915-15925

21. Lynd LR, Grethlein HE (1987) Hydrolysis of dilute acid pretreated mixed hardwood and purified microcrystalline cellulose by cell-free broth from Clostridium thermocellum. Biotechnol Bioeng 29:92-100. doi:10.1002/bit.260290114

22. Lynd LR, Grethlein HE, Wolkin RH (1989) Fermentation of cellulosic substrates in batch and continuous culture by Clostridium thermocellum. Appl Environ Microbiol 55:3131-3139

23. Lynd LR, Laser MS, Bransby D, Dale BE, Davison B, Hamilton R, Himmel M, Keller M, McMillan JD, Sheehan J, Wyman
CE (2008) How biotech can transform biofuels. Nat Biotechnol 26:169-172. doi:10.1038/nbt0208-169

24. Lynd LR, van Zyl WH, McBride JE, Laser M (2005) Consolidated bioprocessing of cellulosic biomass: an update. Curr Opin Biotechnol 16:577-583. doi:10.1016/j.copbio.2005.08.009

25. Lynd LR, Weimer PJ, van Zyl WH, Pretorius IS (2002) Microbial cellulose utilization: fundamentals and biotechnology. Microbiol Mol Biol R 66:506-577

26. Moat AG, Foster JW, Spector MP (2002) Microbial Physiology, 4th edn. Wiley-Liss Inc., New York

27. Olson DG, Lynd LR (2012) Transformation of Clostridium thermocellum by electroporation. Method Enzymol 510:317-330. doi:10.1016/B978-0-12-415931-0.00017-3

28. Ozkan M, Yilmaz EI, Lynd LR, Ozcengiz G (2004) Cloning and expression of the Clostridium thermocellum L-lactate dehydrogenase gene in Escherichia coli and enzyme characterization. Can J Microbiol 50:845-851. doi:10.1139/w04-071

29. Raman B, McKeown CK, Rodriguez M Jr, Brown SD, Mielenz JR (2011) Transcriptomic analysis of Clostridium thermocellum ATCC 27405 cellulose fermentation. BMC Microbiol 11:134. doi:10.1186/1471-2180-11-134

30. Raman B, Pan C, Hurst GB, Rodriguez M Jr, McKeown CK, Lankford PK, Samatova NF, Mielenz JR (2009) Impact of pretreated Switchgrass and biomass carbohydrates on Clostridium thermocellum ATCC 27405 cellulosome composition: a quantitative proteomic analysis. PLoS One 4:e5271. doi:10.1371/journal. pone.0005271

31. Rydzak T, Grigoryan M, Cunningham ZJ, Krokhin OV, Ezzati P, Cicek N, Levin DB, Wilkins JA, Sparling R (2014) Insights into electron flux through manipulation of fermentation conditions and assessment of protein expression profiles in Clostridium thermocellum. Appl Microbiol Biotechnol 98:6497-6510. doi:10.1007/s00253-014-5798-0

32. Rydzak T, Levin DB, Cicek N, Sparling R (2009) Growth phasedependant enzyme profile of pyruvate catabolism and end-product formation in Clostridium thermocellum ATCC 27405. J Biotechnol 140:169-175. doi:10.1016/j.jbiotec.2009.01.022

33. Rydzak T, Levin DB, Cicek N, Sparling R (2011) End-product induced metabolic shifts in Clostridium thermocellum ATCC 27405. Appl Microbiol Biot 92:199-209. doi:10.1007/ s00253-011-3511-0

34. Rydzak T, McQueen PD, Krokhin OV, Spicer V, Ezzati P, Dwivedi RC, Shamshurin D, Levin DB, Wilkins JA, Sparling R (2012) Proteomic analysis of Clostridium thermocellum core metabolism: relative protein expression profiles and growth phase-dependent changes in protein expression. BMC Microbiol 12:214. doi:10.1186/1471-2180-12-214

35. Shanks RMQ, Kadouri DE, MacEachran DP, O'Toole GA (2009) New yeast recombineering tools for bacteria. Plasmid 62:88-97

36. Shao X, Raman B, Zhu M, Mielenz JR, Brown SD, Guss AM, Lynd LR (2011) Mutant selection and phenotypic and genetic characterization of ethanol-tolerant strains of Clostridium thermocellum. Appl Microbiol Biot 92:641-652. doi:10.1007/ s00253-011-3492-Z

37. Sparling R, Islam R, Cicek N, Carere C, Chow H, Levin DB (2006) Formate synthesis by Clostridium thermocellum during anaerobic fermentation. Can J Microbiol 52:681-688. doi:10.1139/w06-021

38. Taillefer M, Rydzak T, Levin DB, Oresnik IJ, Sparling R (2015) Reassessment of the Transhydrogenase 'Malate Shunt' in Clostridium thermocellum ATCC 27405 through kinetic characterization of malic enzyme and malate dehydrogenase. Appl Environ Microbiol. doi:10.1128/AEM.03360-14

39. Tripathi SA, Olson DG, Argyros DA, Miller BB, Barrett TF, Murphy DM, McCool JD, Warner AK, Rajgarhia VB, Lynd LR, Hogsett DA, Caiazza NC (2010) Development of pyrF-based 
genetic system for targeted gene deletion in Clostridium thermocellum and creation of a pta mutant. Appl Environ Microbiol 76:6591-6599. doi:10.1128/AEM.01484-10

40. Tyurin MV, Desai SG, Lynd LR (2004) Electrotransformation of Clostridium thermocellum. Appl Environ Microbiol 70:883-890

41. van der Veen D, Lo J, Brown SD, Johnson CM, Tschaplinski TJ, Martin M, Engle NL, van den Berg RA, Argyros AD, Caiazza NC, Guss AM, Lynd LR (2013) Characterization of Clostridium thermocellum strains with disrupted fermentation end-product pathways. J Ind Microbiol Biot 40:725-734. doi:10.1007/ s10295-013-1275-5

42. Wilson CM, Yang S, Rodriguez M Jr, Ma Q, Johnson CM, Dice L, Xu Y, Brown SD (2013) Clostridium thermocellum transcriptomic profiles after exposure to furfural or heat stress. Biotechnol Biofuels 6:131. doi:10.1186/1754-6834-6-131

43. Yang S, Giannone RJ, Dice L, Yang ZK, Engle NL, Tschaplinski TJ, Hettich RL, Brown SD (2012) Clostridium thermocellum ATCC27405 transcriptomic, metabolomic and proteomic profiles after ethanol stress. BMC Genom 13:336. doi:10.1186/1471-2164-13-336

44. Zhou J, Olson DG, Argyros DA, Deng Y, van Gulik WM, van Dijken JP, Lynd LR (2013) Atypical glycolysis in Clostridium thermocellum. Appl Environ Microbiol 79:3000-3008. doi:10.1128/AEM.04037-12 